\title{
Validation of Automated Quantification of Myocardial Perfusion Single-Photon Emission Computed Tomography Using Heart Score View in Patients With Known or Suspected Coronary Artery Disease
}

\author{
Toshitaka Iwasaki, ${ }^{1}$ MD, Satoshi Kurisu, ${ }^{1}$ MD, Nobukazu Abe, ${ }^{2}$ MD, Megumi Tamura, ${ }^{2}$ MD, \\ Noriaki Watanabe, ${ }^{1}$ MD, Hiroki Ikenaga, ${ }^{1}$ MD, Takashi Shimonaga, ${ }^{1}$ MD, Tadanao HigaKi, ${ }^{1}$ MD, \\ Ken Ishibashi, ${ }^{1} \mathrm{MD}$, Naoya Mitsuba, ${ }^{1} \mathrm{MD}$, Yoshihiro Dohi, ${ }^{1} \mathrm{MD}$, and Yasuki Kinara, ${ }^{1}$ MD
}

\section{SUMMARY}

Heart Score View (HSV) is a free software package for automated quantification of myocardial single photon emission computed tomography (SPECT) imaging using a standard Windows computer. We compared scoring results of myocardial perfusion among visual analysis, Quantitative Perfusion SPECT (QPS), and HSV in patients with known or suspected coronary artery disease.

This study included 75 consecutive patients with known or suspected coronary artery disease who underwent adenosive stress-rest Tl-201 SPECT. Analysis of myocardial perfusion SPECT was performed on a standard 17-segment model visually and using QPS and HSV.

There were 54 male and 21 female patients with a mean age of $70.5 \pm 10.7$ years. Thirteen patients $(17 \%)$ had prior myocardial infarction. Summed stress score (SSS) and summed rest score (SRS) in the mid and basal areas were significantly higher on HSV than visual analysis or QPS. There was no significant difference in SDS in the whole area among the 3 methods. Similar results were found even in patients without prior myocardial infarction. Manual setting of the left ventricular cavity improved the correlations of SSS, SRS and SDS between HSV and the other methods.

Our data suggested that HSV was comparable with visual analysis or QPS in scoring myocardial perfusion when manual setting of the left ventricular cavity is applied. (Int Heart J 2014; 55: 350-356)

Key words: Myocardial ischemia, Scintigraphy

$\mathrm{M}$ yocardial perfusion single photon emission computed tomography (SPECT) with exercise or pharmacoogical stress is a noninvasive method to detect coronary artery disease. ${ }^{1-3)}$ Although myocardial perfusion SPECT allows accurate diagnosis of coronary artery disease, visual analysis is subject to inter- and intraobserver variability even for experienced observers. To overcome these limitations, several software packages for automated quantification such as Quantitative Perfusion SPECT (QPS; version 7.2) (Cedars-Sinai Medical Center, USA) have been developed and commonly used to optimise reproducibility and improve accuracy. ${ }^{4 \cdot 6} \mathrm{Re}$ cently, a new software package called Heart Score View (HSV; version 1.5) (Nihon Medi-Physics, Japan) was developed in Japan. ${ }^{7-9)}$ In the current study, we compared the scoring results of myocardial perfusion among visual analysis, QPS and HSV in patients with known or suspected coronary artery disease.

\section{MeTHODS}

Patients: This study included 75 consecutive patients with known or suspected coronary artery disease who underwent adenosive stress-rest Tl-201 SPECT at Hiroshima University Hospital. Patients with prior myocardial infaction or prior coronary intervention were included. Patients with significant valvular disease or congestive heart failure were excluded.

Tl-201 SPECT: All patients fasted overnight, and underwent stress-rest Tl-201 gated SPECT. ${ }^{10)}$ Adnosine was infused over 6 minutes $(120 \mu \mathrm{g} / \mathrm{kg} /$ minute), and Tl-201 (111 MBq [3.0 $\mathrm{mCi}]$ ) was injected 3 minutes after the initiation of adenosine infusion. The stress Tl-201 SPECT acquisition was started 5 minutes after the stress test. Four hours later, rest Tl-201 SPECT images were also obtained. ECG-gated myocardial perfusion images were acquired with a dual-detector $90^{\circ} \gamma$-camera (E.CAM; Siemens Medical Solutions). Images were acquired with the following parameters: 32 total projections; $180^{\circ}$ from right anterior oblique to left posterior oblique and a noncircular orbit; $64 \times 64$ matrix; $0.6-\mathrm{cm}$ pixel size; 8

From the Departments of ${ }^{1}$ Cardiovascular Medicine and ${ }^{2}$ Clinical Radiology, Hiroshima University Graduate School of Biomedical and Health Sciences, Hiroshima, Japan.

Address for correspondence: Satoshi Kurisu, MD, 1-2-3, Kasumi-cho, Minami-ku, Hiroshima, 734-8551, Japan. E-mail: skurisu@nifty.com

Received for publication October 15, 2013. Revised and accepted December 27, 2013.

Released in advance online on J-STAGE June 5, 2014.

All rights reserved by the International Heart Journal Association. 
frames per cardiac cycle; low-energy, high-resolution collimation; and 40 seconds per stop. Tl-201 SPECT images were acquired with a $10 \%$ symmetric window over the $80-\mathrm{keV}$ Tl-201 photopeak. Filtered backprojection with a Butterworth prefilter (order, 5; cutoff frequency, 0.65 cycles/pixel for rest and stress images) and decay correction were used for reconstruction. No scatter or attenuation correction was applied.

Visual analysis: Visual analysis of myocardial perfusion SPECT was performed on a standard 17-segment model. ${ }^{11)}$ Each segment was graded by consensus by two nuclear medicine physicians (T.I. and S.K.) using a 5-point scale ( 0 , normal uptake; 1 , mildly reduced uptake; 2 , moderately reduced uptake; 3 , severely reduced uptake; 4 , absence of detectable radiotracer in a segment) recommended by the Cardiac Imaging Committee of the American Heart Association and American Society of Nuclear Cardiology (ASNC). ${ }^{12)}$ The summed stress score (SSS) and the summed rest score (SRS) were obtained by adding the scores for the 17 segments. The summed difference score (SDS) was obtained by subtracting SRS from SSS.

Automated quantification: Automated quantification of myocardial perfusion was performed using QPS (version 7.2) and HSV (version 1.5). The HSV software generates a polar-map from myocardial perfusion SPECT images. Mean \% uptake in each segment is automatically scored from normal (0) to absent (4) using a 5-point scale based on ASNC gudelines. The thresholds of $\%$ uptake for scoring were described in previous reports. Briefly, segments with a mean uptake of $<70 \%$ on images were defined as abnormal. Segments with 60 to $70 \%, 50$ to $60 \%$, and 40 to $50 \%$ uptake were scored as 1,2 , and 3, respectively. Segments with $<40 \%$ uptake were recognized as absent uptake, and scored as 4 . Values of SSS, SRS and SDS on stress-rest Tl-201 gated SPECT were automatically calculated with QPS and HSV using a normal database developed for Japanese patients. ${ }^{13)}$ Reverse redistribution on a rest Tl-201 SPECT image was not reflected in the SDS, and it was considered to be zero. Regional SSS, SRS and SDS in apical, mid and basal areas were also calculated to evaluate the characteristics of each software.

Statistical analysis: All data are expressed as the mean \pm SD. Analysis of variance was performed to test for differences of values among the 3 methods. Multiple comparisons were analyzed using the Bonferroni test. Correlations between the 2 methods were tested with Spearman's rank correlation coefficient $(\rho)$. Bland-Altman plot analysis was applied to assess the agreement between the 2 methods. The regression of the mean and the difference between the 2 methods were analyzed. Differences were considered significant if the $P$ value was $<0.05$.

\section{Results}

Patient characteristics: Patient characteristics are shown in Table I. There were 54 male and 21 female patients with a mean age of $70.5 \pm 10.7$ years. Forty-eight patients $(64 \%)$ had hypertension and $26(35 \%)$ had diabetes mellitus. Thirteen patients $(17 \%)$ had prior myocardial infarction. The infarct-related artery was the left anterior descending artery in 8 patients, left circumflex artery in one patient, and right coronary artery in 4 patients. Thirteen patients $(17 \%)$ had prior coronary intervention.

SSS, SRS and SDS: SSS, SRS and SDS assessed by visual
Table I. Patient Characteristics

\begin{tabular}{lc}
\hline & \\
\hline Number of patients & 75 \\
Male gender & $54(72 \%)$ \\
Age (years) & $70.5 \pm 10.7$ \\
Hypertension & $48(64 \%)$ \\
Diabetes mellitus & $26(35 \%)$ \\
Dyslipidemia & $39(52 \%)$ \\
Prior myocardial infarction & $13(17 \%)$ \\
Prior coronary intervention & $13(17 \%)$ \\
Medications & \\
$\quad$ Angiotensin-converting enzyme inhibitors & $7(9 \%)$ \\
Angiotensin II type 1 receptor blockers & $34(45 \%)$ \\
Beta blockers & $13(17 \%)$ \\
Calcium channel blockers & $7(9 \%)$ \\
Statins & $33(44 \%)$ \\
Aspirin & $28(37 \%)$ \\
\hline
\end{tabular}

Table II. SSS, SRS and SDS Values Assessed by the 3 Methods

\begin{tabular}{lcccc}
\hline & Visual analysis & QPS & HSV & $P$ \\
\hline SSS & $5.9 \pm 7.2^{*}$ & $5.8 \pm 5.9^{*}$ & $10.3 \pm 7.3$ & $<0.01$ \\
SRS & $2.4 \pm 5.9^{*}$ & $3.1 \pm 5.4^{*}$ & $7.2 \pm 7.1$ & $<0.01$ \\
SDS & $3.5 \pm 4.0$ & $2.8 \pm 2.8$ & $3.7 \pm 3.9$ & NS \\
\hline
\end{tabular}

SSS indicates summed stress score; SRS, summed rest score; SDS, summed difference score; QPS, quantitative perfusion single photon emission computed tomography; and HSV, heart score view. " $P<0.01$ versus HSV.

analysis, QPS and HSV are shown in Table II. SSS and SRS were significantly higher on HSV than visual analysis or QPS (Figure 1). There was no significant difference in SDS among the 3 methods.

Regional SSS, SRS and SDS in all the patients are shown in Figure 2. SSS and SRS in the mid and basal areas were significantly higher on HSV than visual analysis or QPS. There was no significant difference in SDS in the whole area among the 3 methods. Similar results were found even in patients without prior myocardial infarction (Figure 3).

Correlations of SSS, SRS and SDS between the different methods: Correlations of SSS, SRS and SDS between the different methods are shown in Table III. In the overall patient population, the best correlations of their values were found between visual analysis and QPS. Correlations between HSV and the other methods were inferior to those between visual analysis and QPS. Similar results were found even in patients without prior myocardial infarction.

Effects of manual setting of HSV on SSS, SRS and SDS: Because SSS and SRS on HSV were higher in the basal area, manual setting of the left ventricular cavity was applied (semiautomated HSV). This manual setting improved the correlations of SSS, SRS and SDS between HSV and the other methods (Table III). There were good correlations of SSS (Figure 4), SRS (Figure 5) and SDS (Figure 6), and Bland-Altman plots showed good agreements of their values between semiautomated HSV and the other methods.

\section{Discussion}

In the current study, we demonstrated the following: 1) 
QPS

Stress
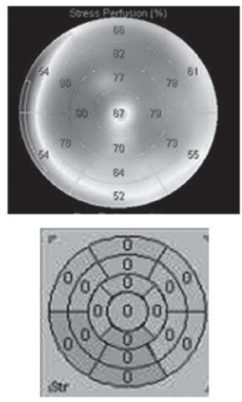

$\mathrm{SSS}=0$
Rest
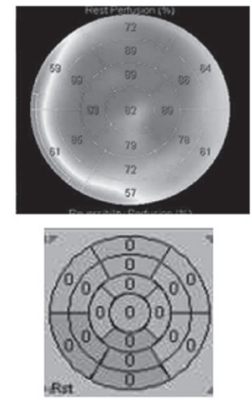

$\mathrm{SRS}=0$
Stress
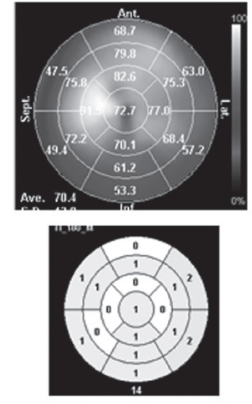

$\mathrm{SSS}=14$
HSV

Rest
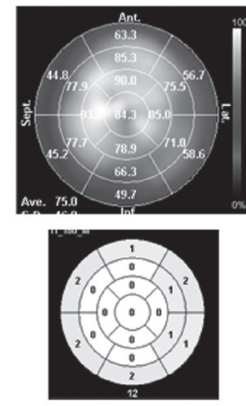

SRS $=12$

Figure 1. Automated quantification using QPS and HSV. In this case, SSS and SRS were markedly higher on HSV than QPS.
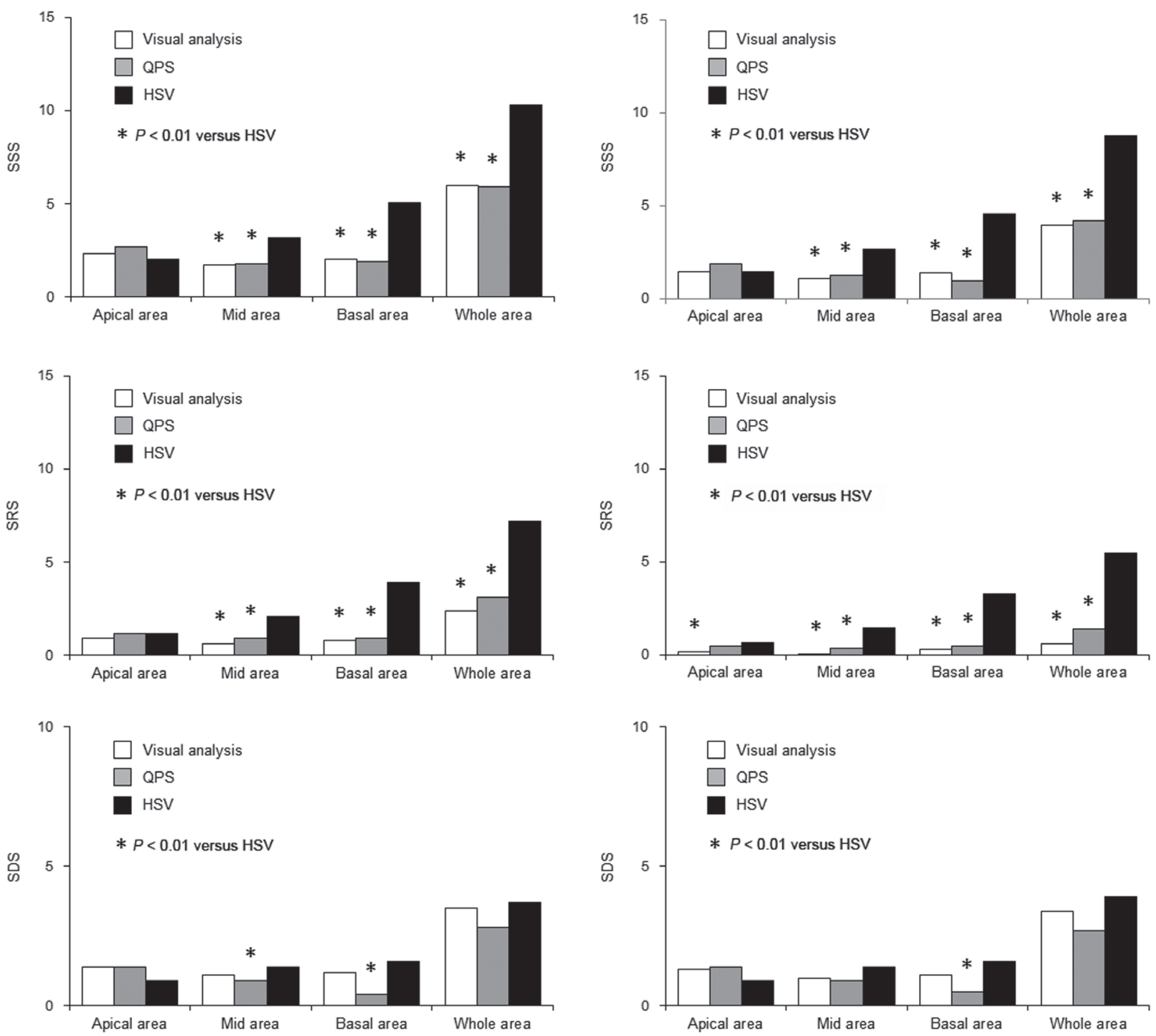

Figure 2. Regional SSS, SRS and SDS in overall patients. SSS indicates summed stress score; SRS, summed rest score ; and SDS, summed difference score.

Figure 3. Regional SSS, SRS and SDS in patients without prior myocardial infarction. SSS indicates summed stress score; SRS, summed rest score; and SDS, summed difference score. 
Table III. Correlations of SSS, SRS and SDS Values Between the 2 Different Methods

\begin{tabular}{|c|c|c|c|c|c|c|c|c|}
\hline & & & \multicolumn{2}{|c|}{ SSS } & \multicolumn{2}{|c|}{ SRS } & \multicolumn{2}{|c|}{ SDS } \\
\hline & & & $\rho$ & $P$ & $\rho$ & $P$ & $\rho$ & $P$ \\
\hline \multicolumn{9}{|c|}{ Overall patients $(n=75)$} \\
\hline Visual analysis & versus & QPS & 0.75 & $<0.01$ & 0.66 & $<0.01$ & 0.77 & $<0.01$ \\
\hline Visual analysis & versus & HSV & 0.67 & $<0.01$ & 0.50 & $<0.01$ & 0.49 & $<0.01$ \\
\hline QPS & versus & HSV & 0.65 & $<0.01$ & 0.43 & $<0.01$ & 0.43 & $<0.01$ \\
\hline Visual analysis & versus & semiautomated HSV & 0.74 & $<0.01$ & 0.50 & $<0.01$ & 0.65 & $<0.01$ \\
\hline QPS & versus & semiautomated HSV & 0.73 & $<0.01$ & 0.52 & $<0.01$ & 0.60 & $<0.01$ \\
\hline \multicolumn{9}{|c|}{ Patients without prior myocardial infarction $(n=62)$} \\
\hline Visual analysis & versus & QPS & 0.68 & $<0.01$ & 0.56 & $<0.01$ & 0.77 & $<0.01$ \\
\hline Visual analysis & versus & HSV & 0.54 & $<0.01$ & 0.30 & $<0.05$ & 0.53 & $<0.01$ \\
\hline QPS & versus & HSV & 0.54 & $<0.01$ & 0.28 & $<0.05$ & 0.50 & $<0.01$ \\
\hline Visual analysis & versus & semiautomated HSV & 0.63 & $<0.01$ & 0.31 & $<0.05$ & 0.66 & $<0.01$ \\
\hline QPS & versus & semiautomated HSV & 0.64 & $<0.01$ & 0.40 & $<0.01$ & 0.62 & $<0.01$ \\
\hline
\end{tabular}

SSS indicates summed stress score; SRS, summed rest score; SDS, summed difference score; QPS, quantitative perfusion single photon emission computed tomography; and HSV, heart score view.
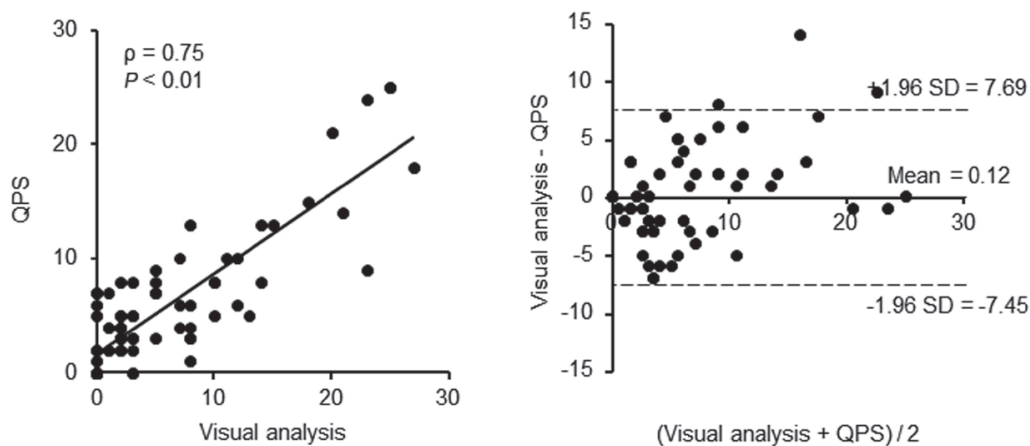

(Visual analysis + QPS)/2
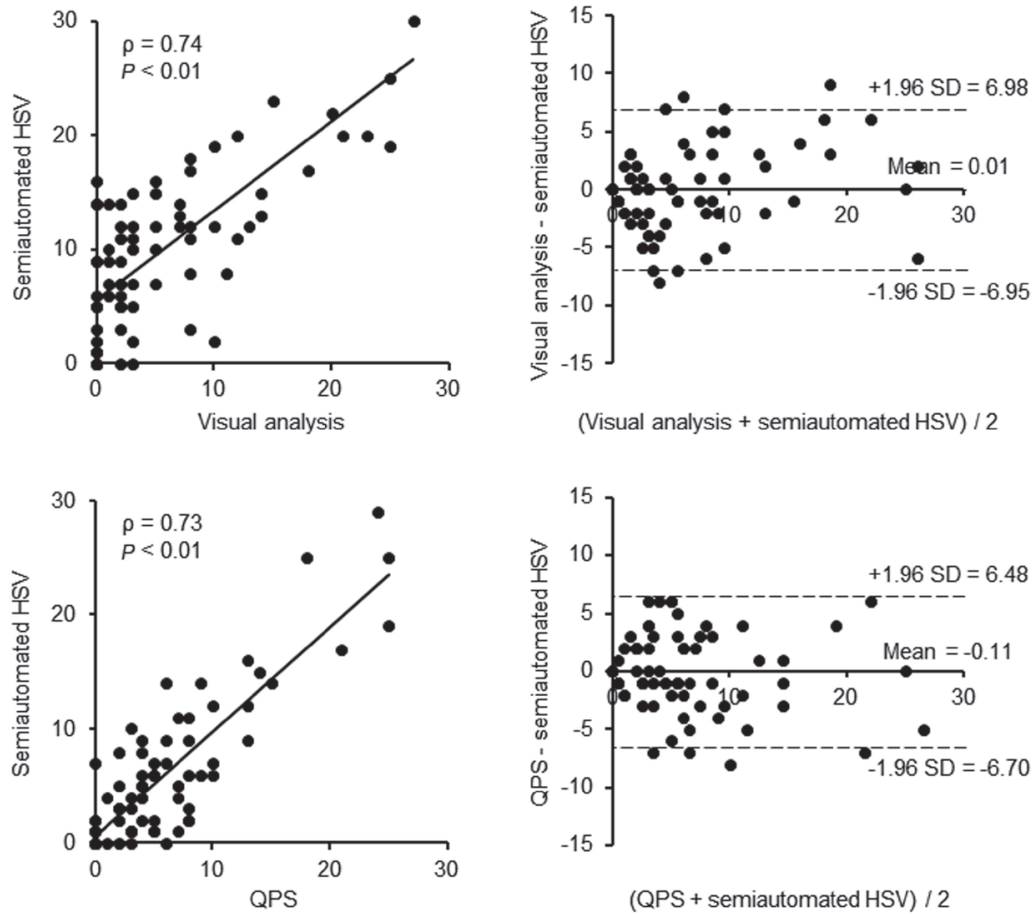

Figure 4. Correlations and scatter plots of SSS between the different methods. SSS indicates summed stress score. 

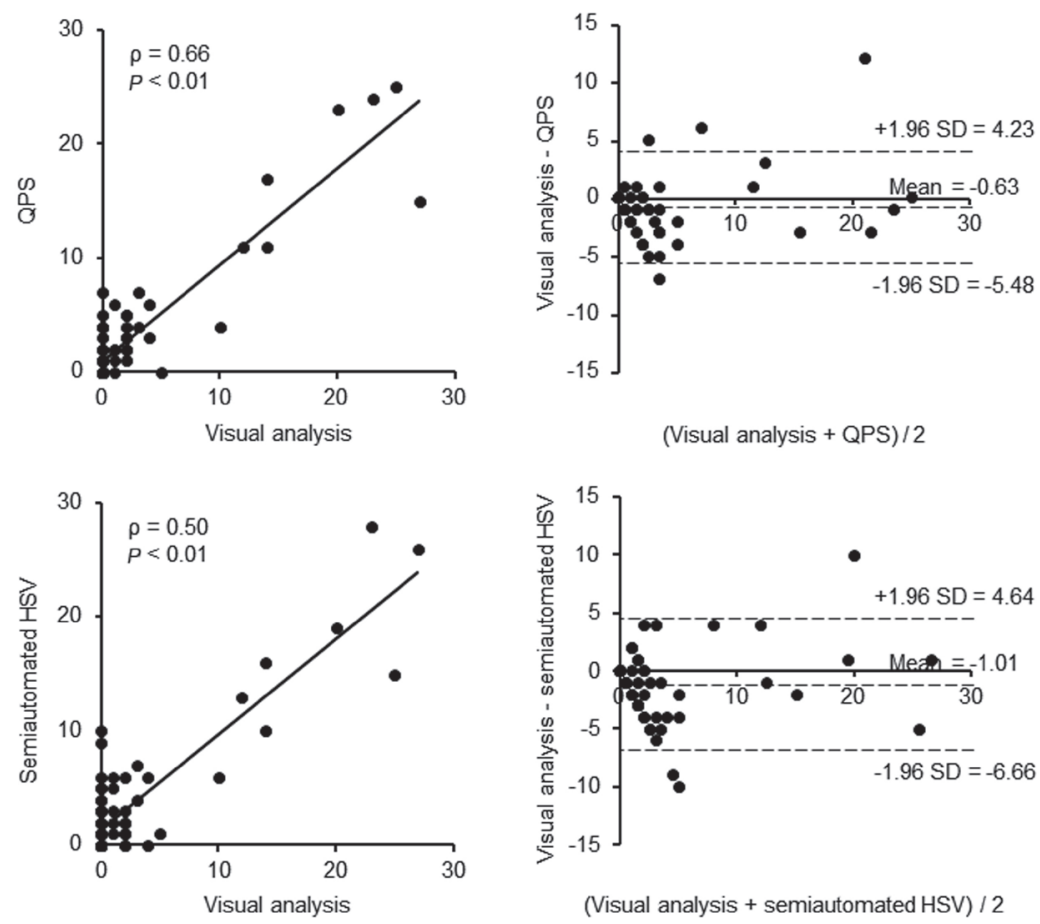

(Visual analysis + QPS)/2
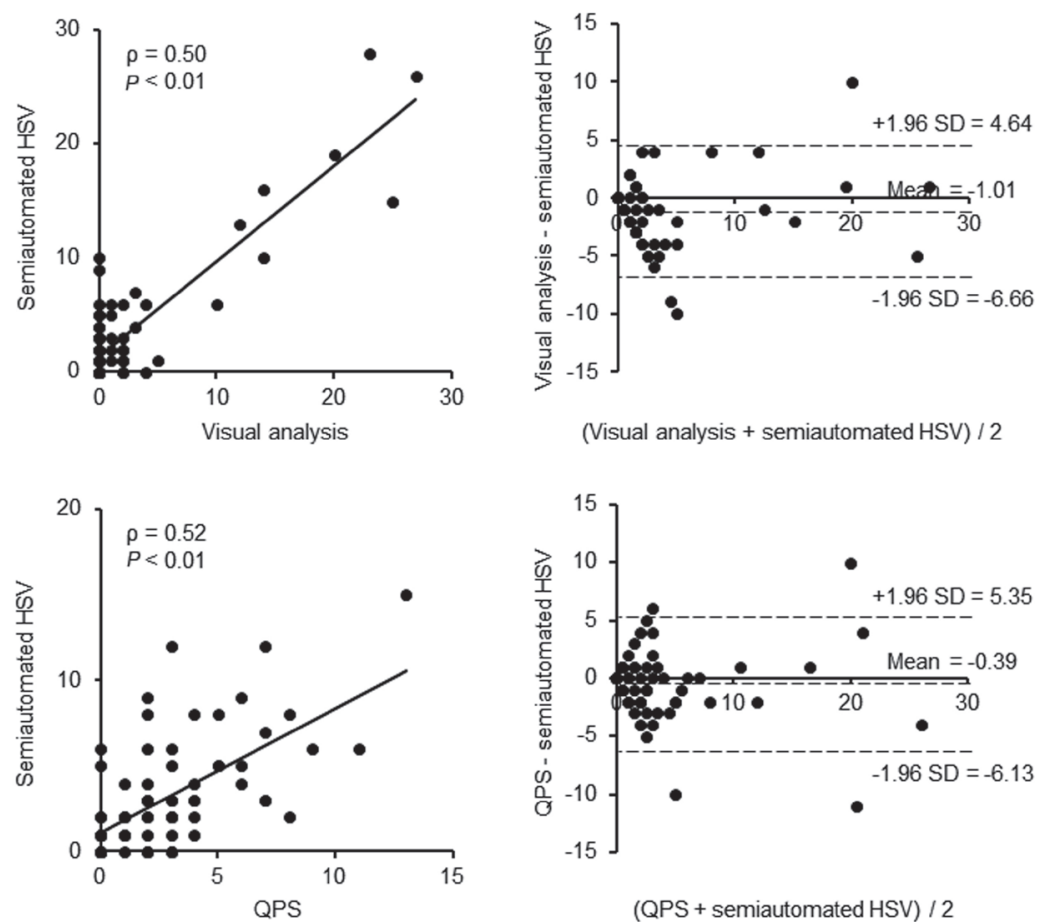

Figure 5. Correlations and scatter plots of SRS between the different methods. SRS indicates summed rest score.

SSS and SRS were significantly higher on HSV than visual analysis or QPS mainly due to different scoring results in the basal area; 2) SDS was similar among the 3 methods; and 3) manual setting of the left ventricular cavity improved the correlations of SSS, SRS and SDS between HSV and the other methods.

Visual analysis of myocardial perfusion SPECT remains to be the standard clinical practice, but is dependent on a subjective interpretation of the data and prone to possible bias related to observer experience. Several software packages for automated quantification such as QPS have been developed. Slomka, et al evaluated the diagnostic performance of QPS in patients with angiographically proven coronary artery disease, and reported that sensitivity, specificity, and accuracy were $92 \%, 79 \%$, and $88 \%$, respectively. ${ }^{2)}$ Nakajima, et al also showed its good diagnostic performance in Japanese patients with coronary artery disease. ${ }^{14)}$ As shown in the current study, SSS, SRS or SDS on QPS correlates well with that on visual analysis. QPS provides high diagnostic performance for the detection of coronary artery disease, and its usefulness is wellestablished.

HSV is a free software package which was recently developed in Japan. ${ }^{7-9)}$ It is applicable to any type of myocardial SPECT imaging using a standard Windows computer. In addition, HSV has another advantage that SPECT data can be analyzed in any institution because HSV is a free software package not requiring a dedicated computer. Several studies have reported the usefulness of HSV in detecting coronary artery disease $^{7,8)}$ or assessing risk stratification, ${ }^{9)}$ but its scoring performance remains to be investigated. In the current study, we showed that SSS and SRS were significantly higher on HSV than visual analysis or QPS mainly due to different scoring results in the basal area. Because HSV systematically produced higher values of SSS and SRS in the basal area, its algorithm appeared to be associated with this phenomenon. Manual setting of the left ventricular cavity resulted in good correlation and agreement of the SSS, SRS or SDS value between HSV and the other methods. Yoshinaga, et al previously evaluated 

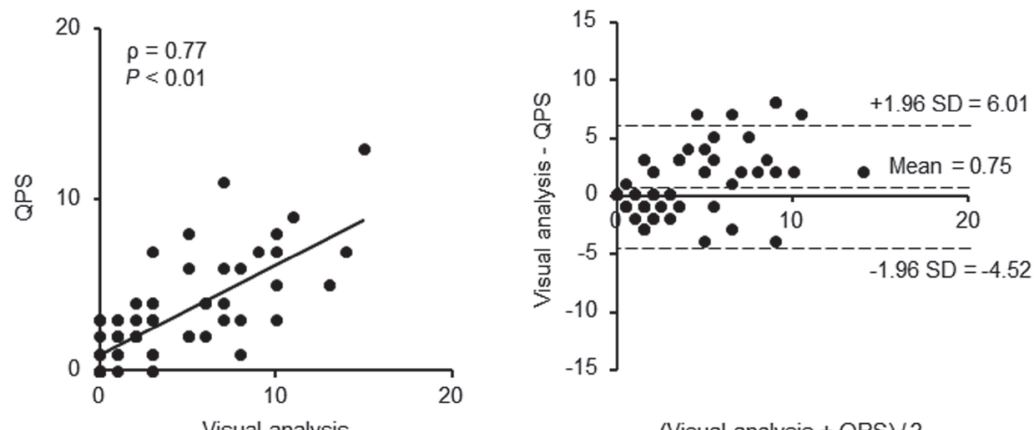

Visual analysis

(Visual analysis + QPS)/2
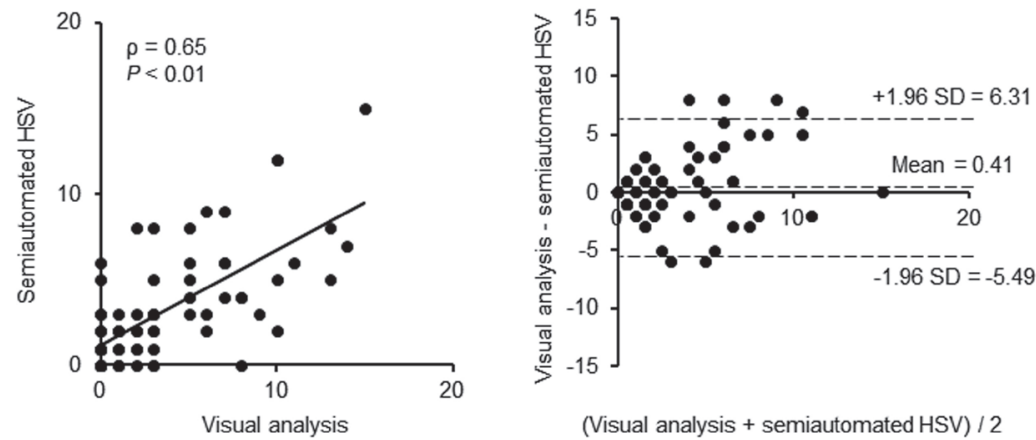

(Visual analysis + semiautomated HSV) $/ 2$
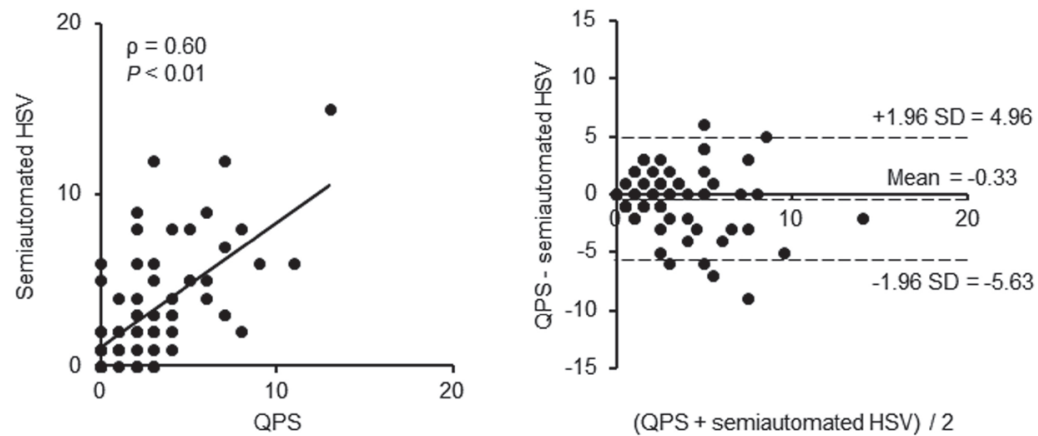

(QPS + semiautomated HSV) /2

Figure 6. Correlations and scatter plots of SDS between the different methods. SDS indicates summed difference score.

the scoring results of HSV with manual setting in patients without prior myocardial infarction, and reported that there were good correlations of these values between visual analysis and HSV. ${ }^{7)}$ Nanasato, et al evaluated the diagnostic performance of HSV in patients with angiographically proven coronary artery disease, and reported that the sensitivity, specificity, and accuracy were $81 \%, 80 \%$, and $80 \%$, respectively. ${ }^{8)}$ According to our results and previous reports, HSV appeared to be comparable with visual analysis or QPS in diagnostic performance of coronary artery disease when manual setting of the left ventricular cavity was applied.

There are some limitations in this study. First, only half of our patients underwent coronary angiography, and we could not show the diagnostic accuracy of detecting the location of myocardial ischemia based on angiographic data. Second, HSV was validated on Tl-201 SPECT. We did not evaluate the reliability of HSV on SPECT using a technetium-labeled tracer. Finally, the small sample size was a major limitation of this study.
In conclusion, HSV is comparable with visual analysis or QPS in scoring myocardial perfusion when manual setting of the left ventricular cavity is applied.

\section{REFERENCES}

1. Akalın EN, Yaylalı O, Kıraç FS, Yüksel D, Kılıç M. The Role of Myocardial Perfusion Gated SPECT Study in Women with Coronary Artery Disease: A Correlative Study. Mol Imaging Radionucl Ther 2012; 21: 69-74.

2. Slomka PJ, Nishina H, Berman DS, et al. Automated quantification of myocardial perfusion SPECT using simplified normal limits. J Nucl Cardiol 2005; 12: 66-77.

3. Chen GB, Wu H, He XJ, et al. Adenosine stress thallium-201 myocardial perfusion imaging for detecting coronary artery disease at an early stage. J Xray Sci Technol 2013; 21: 317-22.

4. Berman DS, Kang X, Gransar H, et al. Quantitative assessment of myocardial perfusion abnormality on SPECT myocardial perfusion imaging is more reproducible than expert visual analysis. $\mathbf{J}$ 
Nucl Cardiol 2009; 16: 45-53.

5. Xu Y, Hayes S, Ali I, et al. Automatic and visual reproducibility of perfusion and function measures for myocardial perfusion SPECT. J Nucl Cardiol 2010; 17: 1050-7.

6. Arsanjani R, Xu Y, Hayes SW, et al. Comparison of fully automated computer analysis and visual scoring for detection of coronary artery disease from myocardial perfusion SPECT in a large population. J Nucl Med 2013; 54: 221-8.

7. Yoshinaga K, Matsuki T, Hashimoto A, Tsukamoto K, Nakata T, Tamaki N. Validation of automated quantitation of myocardial perfusion and fatty acid metabolism abnormalities on SPECT images. Circ J 2011; 75: 2187-95.

8. Nanasato M, Morita S, Yoshida R, et al. Detection of coronary artery disease using automated quantitation of myocardial perfusion on single-photon emission computed tomography images from patients with angina pectoris without prior myocardial infarction. Circ J 2012; 76: 2280-2.

9. Nakata T, Hashimoto A, Matsuki T, Yoshinaga K, Tsukamoto K, Tamaki N. Prognostic value of automated SPECT scoring system for coronary artery disease in stress myocardial perfusion and fatty acid metabolism imaging. Int J Cardiovasc Imaging 2013; 29: 253-62.
10. Matsuki T, Tamaki N, Nakata T, et al. Prognostic value of fatty acid imaging in patients with angina pectoris without prior myocardial infarction: comparison with stress thallium imaging. Eur J Nucl Med Mol Imaging 2004; 31: 1585-91.

11. Holly TA, Abbott BG, Al-Mallah M, et al; American Society of Nuclear Cardiology. Single photon-emission computed tomography. J Nucl Cardiol 2010; 17: 941-73.

12. Cerqueira MD, Weissman NJ, Dilsizian V, et al; American Heart Association Writing Group on Myocardial Segmentation and Registration for Cardiac Imaging. Standardized myocardial segmentation and nomenclature for tomographic imaging of the heart. A statement for healthcare professionals from the Cardiac Imaging Committee of the Council on Clinical Cardiology of the American Heart Association. J Nucl Cardiol 2002; 9: 240-5. (Review)

13. Nakajima K, Kumita S, Ishida Y, et al. Creation and characterization of Japanese standards for myocardial perfusion SPECT: database from the Japanese Society of Nuclear Medicine Working Group. Ann Nucl Med 2007; 21: 505-11.

14. Nakajima K, Okuda K, Kawano M, et al. The importance of population-specific normal database for quantification of myocardial ischemia: comparison between Japanese 360 and 180-degree databases and a US database. J Nucl Cardiol 2009; 16: 422-30. 